01

\title{
Сравнение различных методов разделения непрерывных перекрывающихся спектральных линий
}

\author{
(C) В.С. Сизиков, А.В. Лавров \\ Университет ИТМО, \\ 197101 Санкт-Петербург, Россия \\ e-mail: sizikov2000@mail.ru, lavrov@corp.ifmo.ru
}

Поступила в редакцию 23.01.2018 г.

Сравниваются несколько методов разделения (сепарации) непрерывных перекрывающихся спектральных линий. В большинстве методов профиль каждой линии моделируется гауссианом или лоренцианом и обрабатывается суммарный измеренный спектр $z$. Количество $N$ и параметры линий обычно оценивают методом производных, однако дифференцирование зашумленного спектра $z$ связано с большими погрешностями. Для повышения точности дифференцирования предлагается использовать сглаживающие сплайны. В методе фурье-самодеконволюции для разрешения перекрывающихся линий используется аподизация (искусственное усечение интерферограммы), позволяющая разрешить линии, однако за счет значительного уменьшения их ширин. В настоящей работе уменьшение ширин линий ради их разрешения не используется, а восстанавливаются истинные профили линий путем минимизации функционала невязки модифицированным методом координатного спуска с применением способа сужающихся ограничений, а также для сравнения методом Нелдера-Мида. В методе Манойлова параметры линий-пиков определяются по сверткам производных от спектра с отдельными пиками. В этом методе введено также понятие „степень наложения“. В настоящей работе вводится обобщенная степень наложения для случая разных амплитуд, ширин и расстояний соседних линий. Приведены численные иллюстрации.

DOI: $10.21883 /$ OS.2018.06.46071.28-18

\section{Введение}

Одной из актуальных задач спектроскопии является задача о разделении (сепарации) непрерывных перекрывающихся спектральных линий [1-7]. Перекрытие линий может возникнуть из-за их близости или вследствие воздействия на излучающее вещество магнитного или электрического поля, в результате чего каждая линия расщепляется (эффект Зеемана или Штарка) на ряд близких пиков (компонент) и возникает сверхтонкая структура линии. Перекрытие спектральных линий может возникнуть также из-за взаимодействия молекул вещества, что ведет к движению молекул и к доплеровскому уширению линий и, как следствие, к их (частичному) наложению.

В этих случаях необходимо разделить перекрывающиеся линии в спектре некоторым способом. Особенностью данной задачи является то, что линии невозможно разделить технически, даже используя спектрометр с повышенной разрешающей способностью. Технически можно уточнить суммарный профиль линий, но сами линии по отдельности не разрешить (если не менять внешние условия - вещество, его температуру, электромагнитные поля и т.д.). Линии (их профили) можно разделить только математическим (и компьютерным) путем. Данная задача относится к так называемым обратным задачам второго типа (когда в принципе невозможно восстановить искомую функцию без математической обработки) $[8,9]$

\section{Постановка задачи}

Пусть в спектре присутствуют $N$ непрерывных (протяженных) линий с профилями интенсивности $z_{j}(\lambda)$, $j=\overline{1, N}$, где $j-$ номер линии, а $\lambda-$ длина волны. Считаем, что линии, вообще говоря, налагаются друг на друга в той или иной степени. Полагаем, что измеряется суммарный спектр-профиль $z(\lambda)$, равный сумме $N$ линий-компонент:

$$
z(\lambda)=\sum_{j=1}^{N} z_{j}(\lambda)+\delta z, \quad a \leq \lambda \leq b,
$$

где $[a, b]$ - пределы суммарного спектра, а $\delta z-$ шум. ${ }^{1}$

Требуется на основе измеренного суммарного, обычно зашумленного спектра $z(\lambda)$ определить количество $N$ линий-компонент и их профили $z_{j}(\lambda)$. При этом допускается моделирование профилей линий некоторыми функциями (гауссианами, лоренцианами и т.д.) и использование дополнительной (априорной) информации о линиях (начальные приближения параметров линий, диапазоны изменения параметров и т.д.).

\section{Моделирование линий и оценка их количества}

Задача восстановления профилей линий-компонент $z_{j}(\lambda)$ по измеренному суммарному профилю $z(\lambda)$

\footnotetext{
${ }^{1} \mathrm{~B}$ настоящей работе не рассматривается сглаживающее влияние аппаратной функции спектрометра на суммарный спектр. Полагается, что такое влияние устранено [6].
} 
(см. (1)) в общем случае не имеет однозначного решения. Поэтому обычно используют моделирование линий $z_{j}(\lambda)$ некоторыми функциями, адекватно представляющими реальные линии. Мы воспользуемся моделированием линий гауссианами и лоренцианами.

При этом для оценки количества линий $N$ используем известный метод производных суммарного спектра $d^{l} z(\lambda) / d \lambda^{l}$, где обычно $l=\overline{1,4}$, т. е. используются производные от 1-го до 4-го порядков $[1,3,4,6,7]$.

\section{Моделирование линий гауссианами}

В работе [1] впервые предложен метод производных с использованием производной лишь 1-го порядка, а в работах $[3,4,6,7]$ и др. использованы производные до 4-го порядка. Моделируем каждую линию $z_{j}(\lambda)$ гауссианом (гауссовой функцией):

$$
z_{j}(\lambda)=A_{j} \exp \left(-\frac{\left(\lambda-\bar{\lambda}_{j}\right)^{2}}{2 \sigma_{j}^{2}}\right), \quad j=\overline{1, N},
$$

где $A_{j}-$ амплитуда линии, $\bar{\lambda}_{j}-$ координата максимума, $\sigma_{j}^{2}$ - дисперсия, при этом $\tau_{j}=\sqrt{2 \ln 2} \sigma_{j}=1.1773 \sigma_{j}$ полуширина гауссиана по уровню $0.5[10,11]$.

Производные порядков $l=1,2,3,4$ от $z_{j}(\lambda)$ по $\lambda$ равны $($ ср. $[4,6,7])$

$$
\begin{gathered}
z_{j}^{\prime}(\lambda)=-\frac{A_{j}}{\sigma_{j}} \exp \left(-\frac{\left(\lambda-\bar{\lambda}_{j}\right)^{2}}{2 \sigma_{j}^{2}}\right)\left(\frac{\lambda-\bar{\lambda}_{j}}{\sigma_{j}}\right), \\
z_{j}^{\prime \prime}(\lambda)=-\frac{A_{j}}{\sigma_{j}^{2}} \exp \left(-\frac{\left(\lambda-\bar{\lambda}_{j}\right)^{2}}{2 \sigma_{j}^{2}}\right)\left(-\frac{\left(\lambda-\bar{\lambda}_{j}\right)^{2}}{\sigma_{j}^{2}}+1\right), \\
z_{j}^{\prime \prime \prime}(\lambda)=\frac{A_{j}}{\sigma_{j}^{3}} \exp \left(-\frac{\left(\lambda-\bar{\lambda}_{j}\right)^{2}}{2 \sigma_{j}^{2}}\right)\left(-\frac{\left(\lambda-\bar{\lambda}_{j}\right)^{2}}{\sigma_{j}^{2}}+3\right) \\
\times\left(\frac{\lambda-\bar{\lambda}_{j}}{\sigma_{j}}\right), \\
z_{j}^{\mathrm{IV}}(\lambda)=\frac{A_{j}}{\sigma_{j}^{4}} \exp \left(-\frac{\left(\lambda-\bar{\lambda}_{j}\right)^{2}}{2 \sigma_{j}^{2}}\right)\left(\frac{\left(\lambda-\bar{\lambda}_{j}\right)^{4}}{\sigma_{j}^{4}}\right. \\
\left.-6 \frac{\left(\lambda-\bar{\lambda}_{j}\right)^{2}}{\sigma_{j}^{2}}+3\right) .
\end{gathered}
$$

Из формул (3)-(6) следует, что

$$
\begin{aligned}
& z_{j}^{\prime}\left(\bar{\lambda}_{j}\right)=0, \quad z_{j}^{\prime \prime}\left(\bar{\lambda}_{j}\right)=-\frac{A_{j}}{\sigma_{j}^{2}}<0, \\
& z_{j}^{\prime \prime \prime}\left(\bar{\lambda}_{j}\right)=0, \quad z_{j}^{\mathrm{IV}}\left(\bar{\lambda}_{j}\right)=3 \frac{A_{j}}{\sigma_{j}^{4}}>0,
\end{aligned}
$$

т. е. отрицательный экстремум 2-й производной и положительный экстремум 4-й производной соответствуют максимуму $j$-й линии, а нулевое значение 1-й и 3-й производных (при условии, что $z_{j}^{\prime \prime}\left(\bar{\lambda}_{j}\right)<0, z_{j}^{\mathrm{IV}}\left(\bar{\lambda}_{j}\right)>0$ ) также указывают на $j$-ю линию.
Из выражений для $z_{j}^{\prime \prime}\left(\bar{\lambda}_{j}\right)$ и $z_{j}^{\mathrm{IV}}\left(\bar{\lambda}_{j}\right)$ в (7) можно определить $A_{j}$ и $\sigma_{j}$ из системы двух уравнений:

$$
\left.\begin{array}{l}
\frac{A_{j}}{\sigma_{j}^{2}}=-z_{j}^{\prime \prime}\left(\bar{\lambda}_{j}\right), \\
\frac{A_{j}}{\sigma_{j}^{4}}=\frac{1}{3} z_{j}^{\mathrm{IV}}\left(\bar{\lambda}_{j}\right),
\end{array}\right\}
$$

откуда

$$
A_{j}=3 \frac{\left[z_{j}^{\prime \prime}\left(\bar{\lambda}_{j}\right)\right]^{2}}{z_{j}^{\mathrm{IV}}\left(\bar{\lambda}_{j}\right)}, \quad \sigma_{j}=\sqrt{3 \frac{-z_{j}^{\prime \prime}\left(\bar{\lambda}_{j}\right)}{z_{j}^{\mathrm{IV}}\left(\bar{\lambda}_{j}\right)}}, j=\overline{1, N} .
$$

В работе [4] также использованы производные 2-го и 4-го порядков и изложена другая оригинальная методика, согласно которой вычисляются свертки 2-й и 4-й производных с отдельно взятым пиком (линией) типа (2). Это также дает возможность оценить $A_{j}$ и $\sigma_{j}$.

Однако реальный (не модельный) измеренный спектр обычно зашумлен и его численное (не по аналитическим формулам (3)-(6)) дифференцирование выполняется со значительными погрешностями [7]. Можно отфильтровать шумы, например фильтром Савицкого-Голея с помощью $m$-функции sgolayfilt.m в системе MatLab [12]. Можно также аппроксимировать спектр сглаживающим сплайном $[13,14]$ и дифференцировать сплайн, как это успешно сделано в работах $[7,15,16]$.

При этом заметим, что на практике невозможно дифференцировать непосредственно линии $z_{j}(\lambda)$, а можно дифференцировать только суммарный спектр $z(\lambda)(1)$. В результате параметры отдельных линий будут определяться по производным суммарного спектра с погрешностями, а ряд линий может вообе не проявиться по некоторым производным согласно критериям (7). Поэтому нужно использовать все производные 1-4-го порядков, а также использовать более точные методы сепарации линий (модифицированный метод координатного спуска [7] и др.), полагая в качестве начальных приближений значения $N, A_{j}, \bar{\lambda}_{j}$ и $\sigma_{j}$, оцененные по методу производных.

\section{Моделирование линий лоренцианами}

Если каждая линия $z_{j}(\lambda), j=\overline{1, N}$, - лоренциан (дисперсионный контур, лоренцева линия) $[6,15,16]$ (cp. (2)):

$$
z_{j}(\lambda)=A_{j} \frac{\tau_{j}^{2}}{\left(\lambda-\bar{\lambda}_{j}\right)^{2}+\tau_{j}^{2}},
$$

где $\tau_{j}$ - полуширина лоренциана по уровню 0.5 , то производные будут равны (ср. (3)-(6))

$$
\begin{gathered}
z_{j}^{\prime}(\lambda)=-2 \frac{A_{j}}{\tau_{j}} \frac{\left(\lambda-\bar{\lambda}_{j}\right) \tau_{j}^{3}}{\left[\left(\lambda-\bar{\lambda}_{j}\right)^{2}+\tau_{j}^{2}\right]^{2}}, \\
z_{j}^{\prime \prime}(\lambda)=-2 \frac{A_{j}}{\tau_{j}^{2}} \frac{\left[\tau_{j}^{2}-3\left(\lambda-\bar{\lambda}_{j}\right)^{2}\right] \tau_{j}^{4}}{\left[\left(\lambda-\bar{\lambda}_{j}\right)^{2}+\tau_{j}^{2}\right]^{3}},
\end{gathered}
$$




$$
\begin{gathered}
z_{j}^{\prime \prime \prime}(\lambda)=2 \frac{A_{j}}{\tau_{j}^{3}} \frac{12\left(\lambda-\bar{\lambda}_{j}\right)\left[\tau_{j}^{2}-\left(\lambda-\bar{\lambda}_{j}\right)^{2}\right] \tau_{j}^{5}}{\left[\left(\lambda-\bar{\lambda}_{j}\right)^{2}+\tau_{j}^{2}\right]^{4}}, \\
z_{j}^{\mathrm{IV}}(\lambda)=2 \frac{A_{j}}{\tau_{j}^{4}} \frac{12\left[\tau_{j}^{4}-10 \tau_{j}^{2}\left(\lambda-\bar{\lambda}_{j}\right)^{2}+5\left(\lambda-\bar{\lambda}_{j}\right)^{4}\right] \tau_{j}^{6}}{\left[\left(\lambda-\bar{\lambda}_{j}\right)^{2}+\tau_{j}^{2}\right]^{5}} .
\end{gathered}
$$

Из формул (10)-(13) следует

$$
\begin{aligned}
& z_{j}^{\prime}\left(\bar{\lambda}_{j}\right)=0, \quad z_{j}^{\prime \prime}\left(\bar{\lambda}_{j}\right)=-2 \frac{A_{j}}{\tau_{j}^{2}}<0, \\
& z_{j}^{\prime \prime \prime}\left(\bar{\lambda}_{j}\right)=0, \quad z_{j}^{\mathrm{IV}}\left(\bar{\lambda}_{j}\right)=24 \frac{A_{j}}{\tau_{j}^{4}}>0 .
\end{aligned}
$$

Можно найти $A_{j}$ и $\tau_{j}$ из системы двух уравнений

$$
\left.\begin{array}{l}
\frac{A_{j}}{\tau_{j}^{2}}=-\frac{1}{2} z_{j}^{\prime \prime}\left(\bar{\lambda}_{j}\right), \\
\frac{A_{j}}{\tau_{j}^{4}}=\frac{1}{24} z_{j}^{\mathrm{IV}}\left(\bar{\lambda}_{j}\right),
\end{array}\right\}
$$

откуда (ср. (8))

$$
A_{j}=6 \frac{\left[z_{j}^{\prime \prime}\left(\bar{\lambda}_{j}\right)\right]^{2}}{z_{j}^{\mathrm{IV}}\left(\bar{\lambda}_{j}\right)}, \quad \tau_{j}=\sqrt{12 \frac{-z_{j}^{\prime \prime}\left(\bar{\lambda}_{j}\right)}{z_{j}^{\mathrm{IV}}\left(\bar{\lambda}_{j}\right)}}, j=\overline{1, N} .
$$

\section{Сепарация спектральных линий}

Следующий этап - выполнение собственно сепарации (разделения, разрешения) линий-компонент, входящих в суммарный измеренный спектр $z(\lambda)$ (см. (1)). Рассмотрим и сравним несколько методов сепарации.

\section{Метод фурье-самодеконволюции}

В методе фурье-самодеконволюции спектра (Fourier self-deconvolution) [2] для разрешения перекрывающихся линий используется аподизация (про аподизацию см. [17]) - искусственное усечение интерферограммы, по которой с помощью преобразования Фурье вычисляется спектр в фурье-спектрометрах [9]. За счет аподизации ширины линий искусственно уменьшаются (до 5 раз). В результате истинные профили линий-компонент искажаются ради их разрешения.

Далее в настоящей работе приводится пример из [2] с двумя перекрывающимися линиями, обработанный методом фурье-самодеконволюции и для сравнения методами координатного спуска [7] и Нелдера-Мида $[18,19]$. Показывается, что линии можно разрешить, не искажая их профили, например, методами координатного спуска и Нелдера-Мида.

\section{Степень наложения линий}

В работе [4] введен эффективный параметр, характеризующий ситуацию с наложением двух пиков, степень наложения пиков (в наших обозначениях):

$$
D=\frac{\tau}{\Delta}
$$

где $\tau-$ полуширина пиков-линий по уровню 0.5 , а $\Delta-$ расстояние между пиками. Однако формула (16) применима для случая, когда полуширины обоих пиков одинаковы. А для случая, вообще говоря, разных полуширин предлагаем следующую формулу:

$$
D=\frac{\bar{\tau}}{\Delta}
$$

где $\bar{\tau}=\left(\tau_{1}+\tau_{2}\right) / 2-$ средняя полуширина 1 -го и 2-го пиков. Однако формула (17) не учитывает возможного различия амплитуд $A$ пиков и случая, когда число пиков $N$ больше двух. Предлагается следующая формула для случае, вообще говоря, разных амплитуд $A_{j}, j=\overline{1, N}$, пиков и $N \geq 2$ :

$$
D_{j, j+1}=\frac{\bar{\tau}_{j, j+1}}{\Delta_{j, j+1}}\left(1+\frac{\left|A_{j}-A_{j+1}\right|}{A_{j}+A_{j+1}}\right), \quad j=\overline{1, N-1},
$$

где $\bar{\tau}_{j, j+1}=\left(\tau_{j}+\tau_{j+1}\right) / 2-$ средняя полуширина соседних $(j$ и $j+1)$ пиков, а $\Delta_{j, j+1}=\bar{\lambda}_{j+1}-\bar{\lambda}_{j}-$ расстояние между соседними пиками.

Сделаем некоторый анализ формул (16)-(18). Если амплитуды линий одинаковы: $A_{j}=A_{j+1}$, то формула (18) переходит в формулу $D_{j, j+1}=\bar{\tau}_{j, j+1} / \Delta_{j, j+1}$, а при $N=2-$ в формулу (17). А если $A_{j+1} \ll A_{j}$, то $D_{j, j+1}=2 \bar{\tau}_{j, j+1} / \Delta_{j, j+1}$. Это говорит о том (и формула (18) это подтверждает), что чем шире пики, а также чем они ближе друг к другу и чем больше отличаются их амплитуды, тем больше степень наложения пиков $D$, a значит, тем сложнее разделить пики при решении обратной задачи (см. далее).

Будем использовать также следующий параметр:

$$
\bar{D}=\frac{1}{N-1} \sum_{j=1}^{N-1} D_{j, j+1}
$$

- среднее значение степени наложения всех $N$ пиковлиний.

Следует отметить также метод генетических алгоритмов [19], метод ,полуслепой“ деконволюции [5], способ моделирования (вычислительных экспериментов) [6] довольно сложные методы разложения суммарного спектра на составляющие. В настоящей работе предлагается более простая методика, включающая дифференцирование суммарного спектра, использование сглаживающего сплайна и минимизацию функционала. 

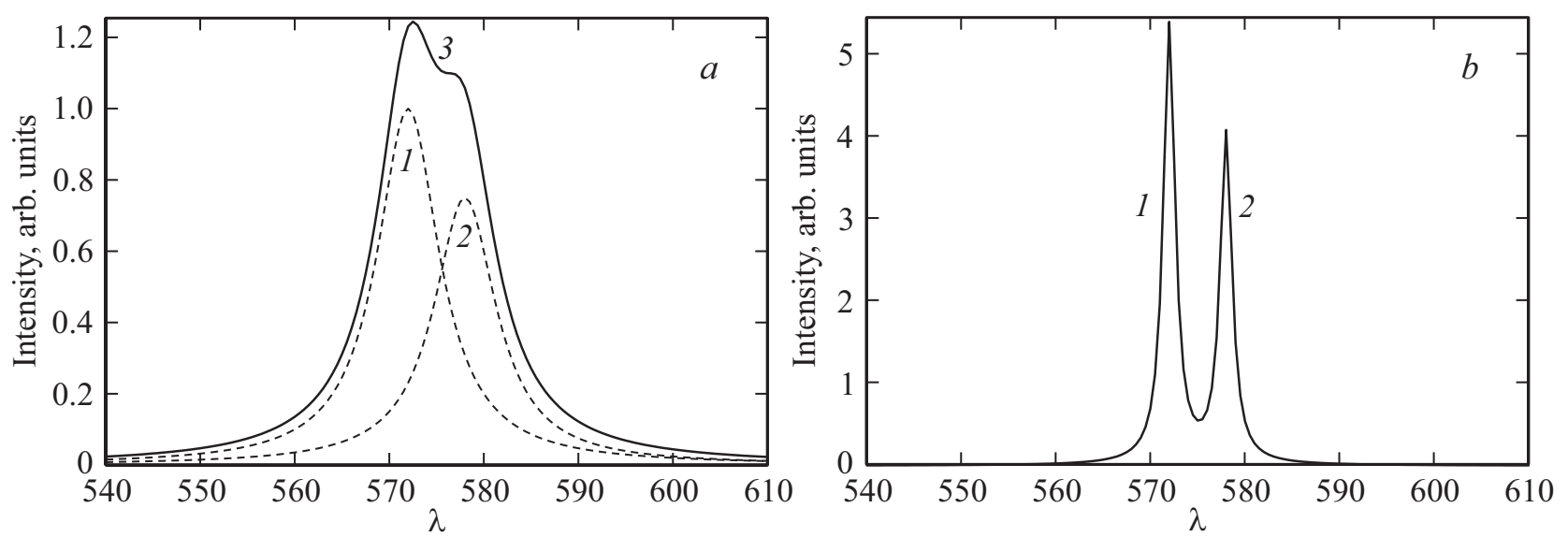

Рис. 1. (a) 1,2 - исходные профили линий $z_{1}(\lambda)$ и $z_{2}(\lambda) ; 3$ - суммарный спектр $z(\lambda)=z_{1}(\lambda)+z_{2}(\lambda) ;(b)$ суммарный спектр $z(\lambda)$, полученный в результате применения метода ФСД, включая аподизацию.

\section{Численные иллюстрации}

В работе [6] нами приведены результаты обработки трех близких линий-гауссиан со средней степенью наложения $\bar{D} \approx 0.52-0.72$. Суммарный спектр $z(\lambda)$ был практически незашумлен, поэтому вычисление 1-й и 2-й производных по $z(\lambda)$ не потребовало использования сплайна. Уточненные параметры трех гауссиан $A_{j}, \bar{\lambda}_{j}$ и $\sigma_{j}$ были найдены путем минимизации функционала невязки модифицированным методом координатного спуска (КС) с ограничениями на параметры, а также (для сравнения) симплекс-методом Нелдера-Мида $(\mathrm{HM})$ с использованием начальных приближений для параметров. Среднеквадратическая погрешность восстановления линий методом КС получилась равной $\delta p \approx 0.011$, а методом НM $-\delta p \approx 0.028$ [6].

С ростом степени наложения $\bar{D}$ производные $z^{\prime}(\lambda)$ и $z^{\prime \prime}(\lambda)$ выделяют не все три линии, но методы КС и НМ их восстанавливают.

В работе [7] приведены результаты обработки также трех линий-гауссиан с $\bar{D} \approx 0.55-0.77$, причем с зашумлением суммарного спектра $z(\lambda)$ 5-процентным шумом. Из-за шума 1-я и 2-я производные от $z(\lambda)$ дали очень неустойчивые результаты, а аппроксимация зашумленного спектра $z(\lambda)$ сглаживающим кубическим сплайном и последующее дифференцирование сплайна позволили оценить число линий $N$ и их координаты $\bar{\lambda}_{j}$. Затем параметры линий $A_{j}, \bar{\lambda}_{j}$ и $\sigma_{j}$ были найдены путем минимизации функционала методами КС (погрешность $\delta p \approx 0.0075-0.043)$ и НМ $(\delta p \approx 0.045-0.072)$.

Эти результаты продемонстрировали эффективность применения сплайн-сглаживания для устойчивого вычисления производных от суммарного спектра $z(\lambda)$ и эффективность определения параметров линий путем минимизации функционала.

В настоящей работе мы сравним результаты сепарации (разделения) линий-компонент методом фурьесамодеконволюции (ФСД) [2], алгоритмом сверток с производными (АСП) [4] и методом минимизации функционала невязки (МФН) [6,7], развиваемым в настоящей работе.

Рассмотрим один из примеров, приведенных в работе [2]. Даны две перекрывающиеся линии-лоренциана вида (9). Амплитуды линий равны $A_{1}=1, A_{2}=0.75$, координаты максимумов $\bar{\lambda}_{1}=572, \bar{\lambda}_{2}=578$, полуширины линий $\tau_{1}=\tau_{2}=8 / 2=4$, а расстояние между пиками $\Delta=\bar{\lambda}_{2}-\bar{\lambda}_{1}=6$. Степень наложения пиков, согласно (19), равна $\bar{D}=0.76$. Спектр задан численно с шагом дискретизации $h=0.5$ в пределах по $\lambda$ : левый предел $a=540$, правый предел $b=610$. Число узлов по $\lambda$ равно $n=(b-a) / h+1=141$ (рис. $1, a)$. Все величины в произвольных единицах, отличных от единиц в [2], где вместо $\lambda$ использовано $1 / \lambda-$ волновое число в $\mathrm{cm}^{-1}$, но графически пример как в [2].

Из рис. $1, b$ видно, что метод ФСД разделил линии, определил их координаты $\bar{\lambda}_{1}$ и $\bar{\lambda}_{2}$ (точнее, расстояние между ними $\Delta=\bar{\lambda}_{2}-\bar{\lambda}_{1}$ ), но занизил (в 5.3 раза) их полуширины $\tau$ (с 4 до 0.75 ), т. е. исказил профили линий.

Используем для сравнения метод МФН. Добавим к суммарному спектру 1-процентный шум $(\delta z=0.005)$, меньше, чем в работе [7] (рис. 2,a).

Используем метод производных, а именно вычислим численно (с помощью $m$-функции diff.m системы MatLab) производные $z^{\prime}(\lambda), z^{\prime \prime}(\lambda), z^{\prime \prime \prime}(\lambda)$ и $z^{\mathrm{IV}}(\lambda)$ от незашумленного и зашумленного суммарного спектра $z(\lambda)$.

На рис. $2, b$ представлены производные $z^{\prime}(\lambda), z^{\prime \prime}(\lambda)$, $z^{\prime \prime \prime}(\lambda)$ и $z^{\mathrm{IV}}(\lambda)$ от незашумленного суммарного спектра $z(\lambda)$. Видим, что кривые в общем соответствуют соотношениям (14). Кривая $z^{\prime}(\lambda)$ имеет два нуля, кривая $z^{\prime \prime}(\lambda)$ имеет два отрицательных экстремума, кривая $z^{\mathrm{IV}}(\lambda)$ имеет два положительных экстремума, причем нули и экстремумы дают значения $\bar{\lambda}_{1}=571.5$ и $\bar{\lambda}_{2}=577.5$, близкие точным значениям $\bar{\lambda}_{1}=572$ и $\bar{\lambda}_{2}=578$. Кривая $z^{\prime \prime \prime}(\lambda)$ имеет слишком много нулей $(>5)$, но надо учитывать лишь те нули, где функция $z^{\prime \prime \prime}(\lambda)$ возрастает, 

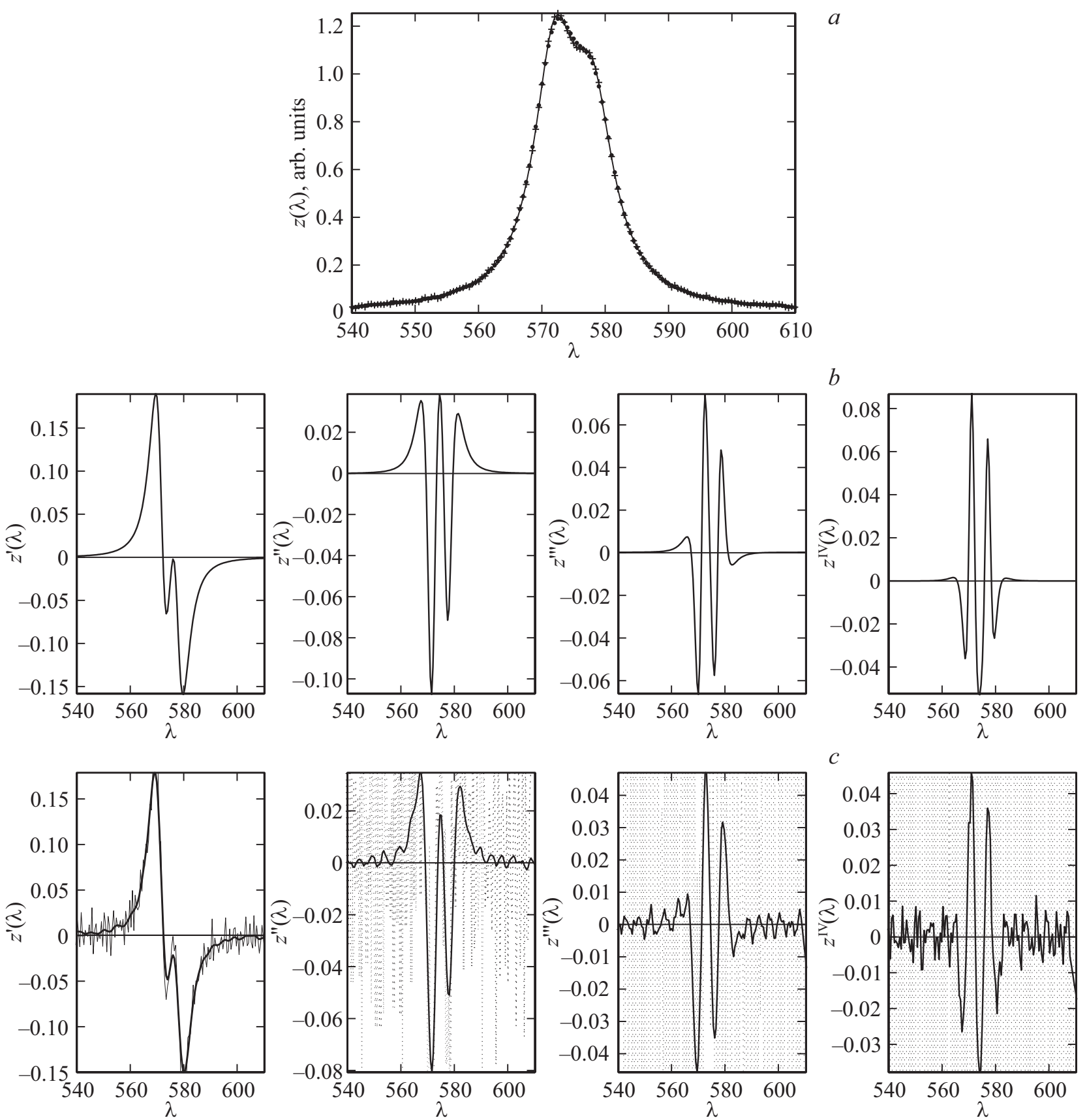

Pис. 2. (a) Суммарный спектр $z(\lambda)$ незашумленный (непрерывная линия), зашумленный (плюсы) и сглаживающий сплайн (точки); $(b)$ производные $z^{\prime}(\lambda), z^{\prime \prime}(\lambda), z^{\prime \prime \prime}(\lambda)$ и $z^{\mathrm{IV}}(\lambda)$ от незашумленного суммарного спектра $z(\lambda) ;(c)$ производные от зашумленного $z(\lambda)$ (тонкие линии, ,пилообразные“ решения) и от сплайна (непрерывные линии).

другими словами, $z^{\prime \prime \prime}(\bar{\lambda})<0$ и $z^{\mathrm{IV}}(\bar{\lambda})>0$ (см. (14)). Таких нулей два.

По рис. 2, $b$ можно в соответствии с формулами (15) оценить значения $A_{1}, \tau_{1}, A_{2}$ и $\tau_{2}$. Из кривых $z^{\prime \prime}(\lambda)$ и $z^{\mathrm{IV}}(\lambda)$ при $\bar{\lambda}_{1}=571.5, \bar{\lambda}_{2}=577.5$ получаем $z^{\prime \prime}\left(\bar{\lambda}_{1}\right)=-0.107$, $z^{\mathrm{IV}}\left(\bar{\lambda}_{1}\right)=0.0689, \quad z^{\prime \prime}\left(\bar{\lambda}_{2}\right)=-0.0713, \quad z^{\mathrm{IV}}\left(\bar{\lambda}_{2}\right)=0.0531$. Используя (15), вычисляем

$$
A_{1}=1.0039, \tau_{1}=4.3246, A_{2}=0.5752, \tau_{2}=4.0154 .
$$

Значения (20) заметно отличаются (особенно $A_{2}$ ) от точных значений

$$
A_{1}=1, \quad \tau_{1}=4, \quad A_{2}=0.75, \quad \tau_{2}=4,
$$

что объясняется (как было сказано выше) тем, что дифференцируется только суммарный спектр $z(\lambda)$, а непосредственно линии $z_{j}(\lambda)$ невозможно дифференцировать в силу их недоступности. Это порождает погрешности в определении параметров линий даже в отсутствие 
шумов. Чтобы уточнить параметры линий, необходимо использовать дополнительно некоторый метод.

Уточнение параметров линий. Для уточнения параметров линий используем модификацию метода координатного спуска (КС) [6,7] (в работе [7] приведен псевдокод этого метода, однако в настоящей работе внесены дополнения в метод КС). Согласно методу КС, компоненты $z_{j}(\lambda), j=\overline{1, N}$, суммарного спектра $z(\lambda)$ моделируем гауссианами (см. (2)) или лоренцианами (см. (9)), у каждой линии по три искомых параметра: амплитуда $A_{j}$, координата максимума $\bar{\lambda}_{j}$ и полуширина $\tau_{j}$ (или СКО $\sigma_{j}$ ).

Далее $3 N$ параметров (где $N$ - число линийкомпонент) объединяем в один вектор

$$
\mathbf{p}=\left[A_{1}, \bar{\lambda}_{1}, \tau_{1}, \ldots, A_{N}, \bar{\lambda}_{N}, \tau_{N}\right]
$$

длиной $3 N$ (в нашем примере $N=2$ ). Параметры $p_{J}$, $J=\overline{1,3 N}$, находим путем минимизации функционала невязки между результатами измерений и расчета:

$$
F=\sum_{i=1}^{n}\left(\tilde{z}_{i}-z_{i}\right)^{2},
$$

где $\tilde{z}_{i}=\tilde{z}\left(\lambda_{i}\right)$ - измеренные значения суммарного спектра, $z_{i}=z_{i}(\mathbf{p})$ - рассчитанные значения суммарного спектра, $n$ - число дискретных отсчетов $\lambda, \mathbf{p}-$ единый вектор (21).

Вводятся также ограничения на параметры $p_{J}$ в виде

$$
p_{\min _{J}} \leq p_{J} \leq p_{\max _{J}}, \quad J=\overline{1,3 N}
$$

Ограничения вида (23) не позволяют выходить решению р за пределы „коридора“, даваемого неравенствами (23), обеспечивая тем самым устойчивость и сходимость решения. При этом предполагается, что ограничения (23) вначале могут быть широкими, а затем могут сужаться - способ сужающихся ограничений.

Для сравнения предлагается выполнить уточнение параметров линий также путем минимизации функционала (22) симплекс-методом Нелдера-Мида (НМ) [18,19], реализованным в m-функции fminsearch.m [20]. Специфика метода НМ состоит в том, что он не использует ограничения, а использует начальные приближения параметров.

Погрешность определения параметров линий. Среднеквадратическая относительная погрешность определения параметров линий-компонент методами КС и НМ может быть вычислена по формуле (ср. [6,7]):

$$
\delta p=\left[\frac{1}{3 N} \sum_{J=1}^{3 N} w_{J}\left(p_{J}-\bar{p}_{J}\right)^{2}\right]^{1 / 2}
$$

где $p_{J}$ - вычисленные значения параметров линий, а $\bar{p}_{J}$ - точные значения параметров (известные лишь при обработке модельных спектров). Здесь $w_{J}-$ веса́, равные, согласно $[6,7], w_{J}=1 / p_{\text {mid } J}^{2}$, причем $p_{\operatorname{mid} J}=\left(p_{\min J}+p_{\max J}\right) / 2$. Однако такое выражение $w_{J}$ годится лишь для метода КС. В настоящей работе предлагается использовать следующее выражение, пригодное как для метода КС, так и для метода НМ: $w_{J}=1 / \bar{p}_{J}^{2}$.

Результаты расчетов. Вначале рассмотрим случай отсутствия шумов (см. рис. 2, $b$ и оценки (20)). Обозначим

$$
\mathbf{p}=[1.0039,571.5,4.3246,0.5752,577.5,4.0154]
$$

вектор вида (21), полученный по производным (см. (20)).

Далее выполняем уточнение вектора р путем минимизации функционала (22) методом КС с использованием сначала довольно широких ограничений вида (23) вокруг значений (20). При этом широкие ограничения $\mathbf{p}_{\min }$ и $\mathbf{p}_{\max }$ вида (23) задаем на основе вектора $\mathbf{p}$ следующим образом (в кодах):

width $=\mathrm{p} ; \quad$ width $(2)=$ width $(5)=10^{*} \mathrm{~h} ; \operatorname{pmin}=\mathrm{p}$ - width; $\mathrm{pmax}=\mathrm{p}+$ width;

или

$$
\begin{aligned}
& p_{\min }(1)=p_{\min }(3)=p_{\min }(4)=p_{\min }(6)=0, \\
& p_{\min }(2)=p(2)-10 h, p_{\min }(5)=p(5)-10 h \text {, } \\
& p_{\max }(1)=2 p(1), \quad p_{\max }(2)=p(2)+10 h, \\
& p_{\max }(3)=2 p(3), \quad p_{\max }(4)=2 p(4), \\
& p_{\max }(5)=p(5)+10 h, \quad p_{\max }(6)=2 p(6), \\
& \text { т.е. (см. (25) и (26)) } \\
& \mathbf{p}_{\min }=[0,566.5,0,0,572.5,0] \text {, } \\
& \left.\mathbf{p}_{\max }=[2.0078,576.5,8.6492,1.1504,582.5,8.0308]\right\}
\end{aligned}
$$

и получаем методом КС уточненный вектор:

$$
\mathbf{p}=[1.0637,572.37,4.4238,0.6374,578.36,3.6686],
$$

$F=0.0119, \delta p=0.0863, N_{f}=1715$ - общее число вызовов функционала.

Видим, что широкие ограничения (27) породили параметры линий p (28), заметно отличные от точных значений (погрешность 8.6\%):

$$
\overline{\mathbf{p}}=[1,572,4,0.75,578,4] .
$$

Для дальнейшего уточнения вектора $\mathbf{p}$ приме́ним способ сужающихся ограничений. Уменьшим ширину ограничений (27) в 1.1 раза:

width $=$ width $/ 1.1 ; \quad$ pmin $=p$-width $; \quad$ max $=p+$ width

(где $\mathrm{p}$ - текущий вектор), после чего вновь выполним минимизацию функционала (22) методом КС. Повторив эти действия в цикле по $i=1, \ldots, k$, получаем

$$
\mathbf{p}=[1.0003,572.00,4.0023,0.7495,578.00,3.9986],
$$


$F=3.11 \cdot 10^{-7}, \delta p=0.00041, N_{f}=23370, k=29$. Цикл по $i$ выполняем, пока

$$
F_{i+1}<F_{i} / 1.001
$$

где $F_{i}$ - значение функционала $F$ в $i$-й итерации, т.е. пока новое значение функционала меньше предыдущего хотя бы на $0.1 \%$.

Метод КС со способом сужающихся ограничений дает параметры линий p, согласно (30), почти совпадающие с точными значениями (29) (погрешность 0.04\%), несмотря на то что используемые исходные значения параметров (20) (или (25)) были весьма далеки от истинных значений.

Выполняем минимизацию функционала (22) также методом НМ, используя в качестве начального приближения вектора р (21) значения (20) (или (25)). Получаем методом НМ (без шумов):

$$
\mathbf{p}=[1.0000,572.00,4.0000,0.7500,578.00,4.0000],
$$

$F=4.13 \cdot 10^{-14}, \delta p=1.06 \cdot 10^{-7}, k=362$. Метод НМ от начальных приближений (25) привел к параметрам (32) - практически точным значениям (29).

Чтобы из полученных решений сделать выбор, помимо критерия (31) предлагается следующий.

Критерий выбора варианта решения:

- если обрабатывается модельный спектр с известными параметрами линий $\overline{\mathbf{p}}$, то отдаем предпочтение варианту с минимальной погрешностью $\delta p$ (согласно (24));

- если обрабатывается реальный спектр с неизвестным $\overline{\mathbf{p}}$, то отдаем предпочтение варианту с минимальным значением функционала невязки $F$ (согласно $(22)$ ).

Исходя из этого критерия в данном примере выбираем решения с минимальной погрешностью $\delta p$.

Теперь рассмотрим случай зашумления суммарного спектра $z(\lambda)$. Из рис. 2, $c$ видно, что зашумление суммарного спектра даже умеренным 1-процентным шумом (рис. $2, a$ ) ведет к большим погрешностям производных $z^{\prime}(\lambda), \quad z^{\prime \prime}(\lambda), \quad z^{\prime \prime \prime}(\lambda)$ и $z^{\mathrm{IV}}(\lambda)-\kappa$ так называемым „пилообразным“ решениям (ср. [21,8,9]), по которым невозможно выделить ограниченное количество нулей производных $z^{\prime}(\lambda)$ и $z^{\prime \prime \prime}(\lambda)$, а также экстремумов производных $z^{\prime \prime}(\lambda)$ и $z^{\mathrm{IV}}(\lambda)$. Такое поведение производных связано с тем, что дифференцирование (тем более высокого порядка) зашумленных функций является некорректной (сильно неустойчивой) задачей [21].

Чтобы сделать вычисление производных устойчивым и умеренно гладким, используем аппроксимацию зашумленного суммарного спектра $z(\lambda)$ сглаживающим кубическим сплайном $[13,14]$ с помощью m-функции csaps.m (с параметром сглаживания $P=0.5)($ cp. $[15,16])$. После сглаживания были вычислены с помощью т-функции diff.m производные $z^{\prime}(\lambda), z^{\prime \prime}(\lambda), z^{\prime \prime \prime}(\lambda)$ и $z^{\mathrm{IV}}(\lambda)$ (рис. $\left.2, c\right)$. Сравнение рис. $2, b$ и $2, c$ показывает, что сплайнпроизводные дают значения $\bar{\lambda}_{1}=571.5$ и $\bar{\lambda}_{2}=578$, почти такие же, как и незашумленные производные (рис. $2, b)$, но значения производных $z^{\prime \prime}\left(\bar{\lambda}_{1,2}\right)$ и $z^{\mathrm{IV}}\left(\bar{\lambda}_{1,2}\right)$ несколько различные.

Со сплайн-кривых $z^{\prime \prime}(\lambda)$ и $z^{\mathrm{IV}}(\lambda)$ на рис. 2,c снимаем при $\bar{\lambda}_{1}=571.5, \bar{\lambda}_{2}=578$ значения: $z^{\prime \prime}\left(\bar{\lambda}_{1}\right)=$ $=-0.0809, \quad z^{\mathrm{IV}}\left(\bar{\lambda}_{1}\right)=0.04336, \quad z^{\prime \prime}\left(\bar{\lambda}_{2}\right)=-0.05114$, $z^{\mathrm{IV}}\left(\bar{\lambda}_{2}\right)=0.02740$. Используя (15), вычисляем (cp. (20))

$$
A_{1}=0.9049, \tau_{1}=4.7307, A_{2}=0.5728, \tau_{2}=4.7329 \text {. }
$$

Параметры (33) заметно отличаются от точных значений и от „бесшумных“ значений (20). Поэтому требуется их уточнение.

Выполняем минимизацию функционала (22) методом КС с использованием „широких“ ограничений вокруг значений (33) по правилу (26):

$$
\left.\begin{array}{l}
\mathbf{p}_{\min }=[0,566.5,0,0,573,0], \\
\mathbf{p}_{\max }=[1.8099,576.5,9.4615,1.1457,583,9.4659] .
\end{array}\right\}
$$

Получаем на основе (34)

$$
\mathbf{p}=[1.0193,572.29,4.4051,0.6501,578.19,3.9534],
$$

$F=0.0171, \delta p=0.0689, N_{f}=1422$. Далее используем способ сужающихся ограничений, в результате получаем

$$
\mathbf{p}=[1.0129,572.04,4.0332,0.740,578.06,3.9353],
$$

$F=0.00263, \quad \delta p=0.0106, \quad N_{f}=14400, \quad k=17 . \quad$ Параметры линий (36) получились более точными (в $0.0689 / 0.0106=6.5$ раз), чем (35), и близкими к точным значениям (29). Это говорит об эффективности способа сужающихся ограничений.

Выполняем минимизацию функционала (22) также методом НМ, используя в качестве начального приближения вектора р (21) значения (33), а именно

$$
\mathbf{p}=[0.9049,571.5,4.7307,0.5728,578,4.7329] .
$$

Получаем методом НМ (с шумами)

$\mathbf{p}=[1.0115,572.0382,4.0262,0.7417,578.0555,3.9427]$,

$F=0.00260, \delta p=0.0092$, число итераций $k=414$. Начальное приближение (37) породило решение (38), дающее значения параметров линий, довольно близкие к точным значениям (29). При этом значения функционала $F$ и погрешности параметров $\delta p$ в методах КС и НМ получились практически одинаковыми.

На рис. 3 представлены линии-лоренцианы, восстановленные методом КС (рис. $3, a$ ) и методом НМ (рис. $3, b$ ) по зашумленным данным, но сглаженным сплайном.

Погрешность $\delta p$ решения методом КС с сужающимися ограничениями получилась равной $1.06 \%$, а методом НМ равной $0.92 \%$, что сопоставимо. Но применение метода КС с многократным сужением ограничений потребовало 14400 оценок целевой функции, а метод НМ использовал 414 оценок целевой функции. 

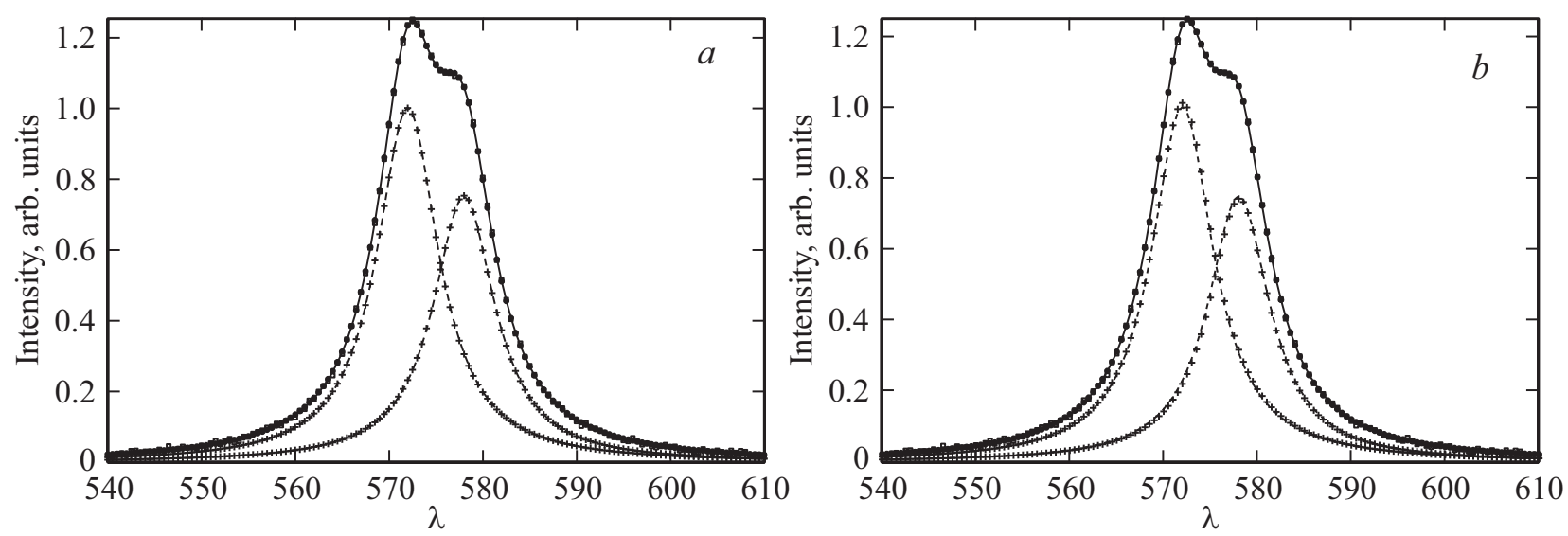

Рис. 3. (a) Восстановление линий-лоренцианов методом КС по данным $z(\lambda)$, зашумленным, но сглаженным сплайном, с использованием способа сужающихся ограничений: непрерывная линия - точный суммарный спектр $z(\lambda)$, две пунктирные линии - точные профили линий с параметрами (29), квадратики - зашумленный суммарный спектр $z(\lambda)$, плюсы - профили двух линий, рассчитанные методом КС; $(b)$ восстановление линий методом НМ, обозначения такие же, как в $(a)$.

Точность результатов метода НМ (и зависящее от этого время выполнения) можно регулировать, задавая порог сходимости целевой функции через параметр optimset m-функции fminsearch.m (в данной paботе результаты получены при задании порога сходимости $\left.10^{-6}\right)$. А точность результатов и время работы метода КС регулируется заданием порога сходимости для каждой из переменных минимизируемой функции (в данной работе использовался порог $10^{-4}$ для всех параметров вектора $p$ ), а также условиями сужения ограничений (здесь использовался коэффициент сужения 1.1 и пороговый коэффициент сходимости функционала 1.001, см. (31)).

\section{Заключение}

1. В работе сопоставлены некоторые методы разделения (сепарации) перекрывающихся спектральных линий, а именно метод производных суммарного спектра, метод фурье-самодеконволюции (ФСД), метод сверток производных с пиками, а также методы координатного спуска (КС) и Нелдера-Мида (НМ) минимизации функционала невязки.

2. Построена следующая цепочка действий в предлагаемой методике разделения линий. Вычисляются численно производные 1-, 2-, 3- и 4-го порядков от, вообще говоря, зашумленного суммарного спектра $z(\lambda)$ с использованием сглаживающего сплайна (рис. 2). По производным делается оценка количества линий $N$ и их координат $\bar{\lambda}_{j}$. Выполняются оценки других параметров линий: амплитуд $A_{j}$ и полуширин $\tau_{j}$ гауссианов согласно (8) или лоренцианов согласно (15) по производным 2-го и 4-го порядков согласно новым выведенным формулам. Далее эти оценки могут быть начальными приближениями при более точном определении параметров линий методами КС и НМ.
3. Предложены новые формулы (18), (19) для степени наложения пиков для случая нескольких пиков, имеющих, вообще говоря, разные ширины и амплитуды.

4. В методе ФСД за счет аподизации удается разделить линии, но при этом ширины линий значительно уменьшаются. Предлагаемая в настоящей работе и в работах $[4,6,7]$ и др. методика восстанавливает истинные профили без их заужения.

5. Рассмотрен численный пример разделения линийлоренцианов методом ФСД и предлагаемой методикой.

\section{Список литературы}

[1] Giese A.T., French C.S. // Appl. Spectrosc. 1955. V. 9. N 2. P. 78. doi 10.1366/000370255774634089

[2] Kauppinen J.K., Moffatt D.J., Mantsch H.H., Cameron D.G. // Appl. Spectrosc. 1981. V. 35. N 3. P. 271. doi 10.1366/0003702814732634

[3] Михайленко В.И., Михальчук В.В. // ЖПС. 1987. Т. 46. № 4. C. 535; Mikhailenko V.I., Mikhal'chuk V.V. // J. Appl. Spectrosc. 1987. V. 46. N 4. P. 327. doi 10.1007/BF00660037

[4] Манойлов В.В., Заруцкий И.В. // Научное приборостроение. 2009. Т. 19. № 4. С. 103.

[5] Yan L., Liu H., Zhong S., Fang H. // Appl. Spectrosc. 2012. V. 66. N 11. P. 1334. doi 10.1366/11-06256

[6] Сизиков В.С., Лавров А.В. // Опт. и спектр. 2017. Т. 123. № 5. C. 678. doi 10.7868/S0030403417110216; Sizikov V.S., Lavrov A.V. // Opt. Spectrosc. 2017. V. 123. N 5. P. 682. doi $10.1134 / \mathrm{S} 0030400 \mathrm{X} 17110200$

[7] Сизиков В.С., Лавров А.В. // Научно-техн. вестник ИТМО. 2017. T. 17. № 5. С. 879 . doi 10.17586/2226-1494-2017-175-879-889; Sizikov V.S., Lavrov A.V. // Sci. Techn. J. ITMO. 2017. V. 17. N 5. P. 879. doi 10.17586/2226-1494-2017-17-5879-889

[8] Сизиков B.C. Обратные прикладные задачи и MatLab. СПб.: Лань, 2011. 256 с.

[9] Сизиков В.С. Прямые и обратные задачи восстановления изображений, спектроскопии и томографии. СПб.: Лань, 2017. $412 \mathrm{c}$. 
[10] Сизиков В.С., Кривых А.В. // Опт. и спектр. 2014. Т. 117. № 6. C. 1040. doi 10.7868/S0030403414110166; Sizikov V.S., Krivykh A.V. // Opt. Spectrosc. 2014. V. 117. N 6. P. 1010. doi 10.1134/S0030400X14110162

[11] Sizikov V., Sidorov D. // Appl. Spectrosc. 2017. V. 71. N 7. P. 1640. doi 10.1177/0003702817694181

[12] Дьяконов В., Абраменкова И. MATLAB. Обработка сигналов и изображений. СПб.: Питер, 2002. 608 с.

[13] Воскобойников Ю.Е., Преображенский Н.Г., Седельников А.И. Математическая обработка эксперимента в молекулярной газодинамике. Новосибирск: Наука, 1984. $240 \mathrm{c}$.

[14] Сизиков В.С. Математические методы обработки результатов измерений. СПб.: Политехника, 2001. 240 с.

[15] Сизиков В.С. // Научно-техн. вестник ИТМО. 2013. № $6(88)$. C. 1.

[16] Sizikov V.S., Evseev V., Fateev A., Clausen S. // Appl. Opt. 2016. V. 55. N 1. P. 208. doi 10.1364/AO.55.000208

[17] Bell R.J. Introductory Fourier Transform Spectroscopy. N.Y.-London: Academic Press, 1972. 382 p.

[18] Nelder J.A., Mead R. // Computer J. 1965. V. 7. N 4. P. 308. doi 10.1093/comjn1/7.4.308

[19] Kincaid D., Cheney $W$. Numerical Analysis: Mathematics of Scientific Computing. 3rd ed. Providence, RI: AMS, 2009. $788 \mathrm{p}$.

[20] Дьяконов В. MATLAB 6 / Учебный курс. СПб.: Питер, 2001. $592 \mathrm{c}$.

[21] Тихонов А.Н., Арсенин В.Я. Методы решения некорректных задач. 3-е изд. М.: Наука, 1986. 288 с. 